\section{Biological-like vesicular structures self-assembled from DNA-block copolymers $\dagger$}

\author{
Nicolas Cottenye, ${ }^{a b}$ Marie-Isabel Syga, ${ }^{a}$ Sergey Nosov, ${ }^{c}$ Axel H. E. Müller, ${ }^{c}$ Lydie Ploux $^{b}$ \\ and Corinne Vebert-Nardin*ad
}

Received 30th November 2011, Accepted 10th January 2012

DOI: $10.1039 / \mathbf{c} 2 \mathrm{cc} 17487 \mathrm{a}$

The polymer modification of short nucleotide sequences has been achieved for future use as self-assembled biologically active structures with sizes in the nanometre range. Co-assembly of the resulting DNA-based amphiphilic block copolymers with native proteins demonstrates the self-assembly of biological-like vesicular structures.

In biology, vesicles are basic yet evolved structures, which are vital to the cell. They are small membrane-enclosed spherical sacks resulting from the supramolecular assembly of phospholipids. They ensure the organization of cellular substances whereas embedding and/or association of proteins enables them to act as chemical reaction chambers involved in digestion of cellular products and wastes, transport, or enzyme storage. Simplified analogous organelles were achieved through co-assembly of synthetic copolymers with native proteins. ${ }^{1}$ Embedding of pore forming bacterial proteins in the synthetic copolymer membrane ensured transport of reaction products and educts across the spherically closed copolymer shell., ${ }^{2,3}$ To achieve a higher level of hierarchical organization and function, copolymers resulting from the coupling of a hydrophobic polymer segment to a nucleotide sequence were recently synthesized to self-assemble in dilute aqueous solution. ${ }^{4,5}$ We demonstrate herein through co-assembly with native proteins that a suitable DNA-block copolymer self-assembles in vesicular structures in dilute aqueous solution. An enzyme is functionally encapsulated in the inner aqueous pool of the self-assembly whereas pore-forming proteins ensure diffusion of the substrate and product of the enzymatic reaction across the copolymer vesicular wall, otherwise not permeable.

The chemical route to the polymer modification of the nucleotide sequences ${ }^{5}$ has been adapted in order to scale up

\footnotetext{
${ }^{a}$ University of Basel, Klingelbergstrasse 80, CH-4056,

Basel, Switzerland.E-mail: nicolas.cottenye@umontreal.ca, isabel.syga@bioss.uni-freiburg.de; Fax: +41(0)6126738 55; Tel: + 41(0)612673800

${ }^{b}$ Institut de Science des Matériaux de Mulhouse, 15, rue Jean Starcky, BP 2488,68057 Mulhouse cedex, France.E-mail: lydie.ploux@uha.fr; Fax: + 33 (3)89608799; Tel: + 33 (3)89608700

${ }^{c}$ Universität Bayreuth, 95440 Bayreuth, Germany.

E-mail: axel.mueller@uni-bayreuth.de,nosovsserge@yahoo.com;

Fax: + 49 (0)921-553393; Tel: + 49 (0)921-553399

${ }^{d}$ University of Geneva - Sciences II, 30, quai Ernest-Ansermet,

CH-1211 Geneva 4, Switzerland.E-mail: corinne.vebert@unige.ch;

Fax: + 41 (0)223796432; Tel: + 41 (0)223796422

$\dagger$ Electronic supplementary information (ESI) available. See DOI: $10.1039 / \mathrm{c} 2 \mathrm{cc} 17487 \mathrm{a}$
}

the synthesis and achieve a relatively larger yield. Model nucleotide sequences, composed of twelve nucleic acids to eventually ensure the formation of a thermodynamically stable double helix upon hybridization through Watson-Crick basepairing, are chosen such that the chain configuration is linear. The synthetic hydrophobic polymers to be grafted to the nucleotide sequence are selected according to their low glass transition temperature to confer sufficient water solubility to the resulting amphiphilic macromolecule and induce non-kinetically frozen self-assembled structures. Either amino-terminated poly (isobutylene) (PIB) or poly(butadiene) (PB) were grafted to nucleotide sequences. We synthesized the following four diblock copolymers: PIB ${ }_{31}$-block-5'-GGGAGAGAGAGA-3' $\left(\mathrm{PIB}_{31}\right.$ $\left.\mathrm{G}_{7} \mathrm{~A}_{5}\right), \mathrm{PB}_{65}$-block-5'-AGAGAGAGAGGG-3' $\left(\mathrm{PB}_{65}-\mathrm{A}_{5} \mathrm{G}_{7}\right)$, $\mathrm{PB}_{65}$-block-5'-CCTCTCTCTCT-3' $\left(\mathrm{PB}_{65}-\mathrm{T}_{5} \mathrm{C}_{7}\right)$ and $\mathrm{PIB}_{31^{-}}$ block-5'-TCTCTCTCTCCC-3' $\left(\mathrm{PIB}_{31}-\mathrm{C}_{7} \mathrm{~T}_{5}\right)$. To prevent tedious synthesis and purification steps, the coupling between the nucleotide sequence functionalized with a carboxylic acid group at the $5^{\prime}$-terminus and the amino-terminated synthetic polymer segment is performed through solid phase synthesis. ${ }^{5}$ Grafting between the DNA fragment and the polymer segment is thus conducted prior to the cleavage from the controlled pore glass (CPG) resin. The signal at $175 \mathrm{ppm}$ in ${ }^{13} \mathrm{C}$-CP-MAS (Fig. 1) assigned to the amide linker evidences the successful coupling of the nucleotide sequence to the synthetic polymer segment through an amide bond. Combined ${ }^{1} \mathrm{H}-\mathrm{NMR}$ (ESI $\dagger$, Fig. S1) and solvent free bulk analyses (ESI $\dagger$, Fig. S2 for FTIR) enable the full assignment of the characteristic peaks of the chemical groups along both the polymer segment and the nucleotide sequence.

Agreement between the theoretical and experimental nitrogen to carbon ratio ( 28.22 and $24.80 \%$, respectively) as assessed by elementary analysis indicates that sequential dialysis and size exclusion chromatography subsequent to solid phase synthesis yields polymer-modified nucleotide sequences of high purity. Since the exact copolymer solution concentration can be determined by UV spectroscopy ( $260 \mathrm{~nm}$ ), the synthesis yield is thus accurately evaluated. From batch to batch, 15 to $30 \%$ efficiency could be achieved. Slow diffusion of the synthetic polymer segment through the pores of the solid support is probably the limiting factor to a higher yield through this chemical route.

As assessed by circular dichroism (CD), neither the coupling of the polymer segment to the nucleotide sequence nor the chemical composition of the synthetic polymer segment affects 


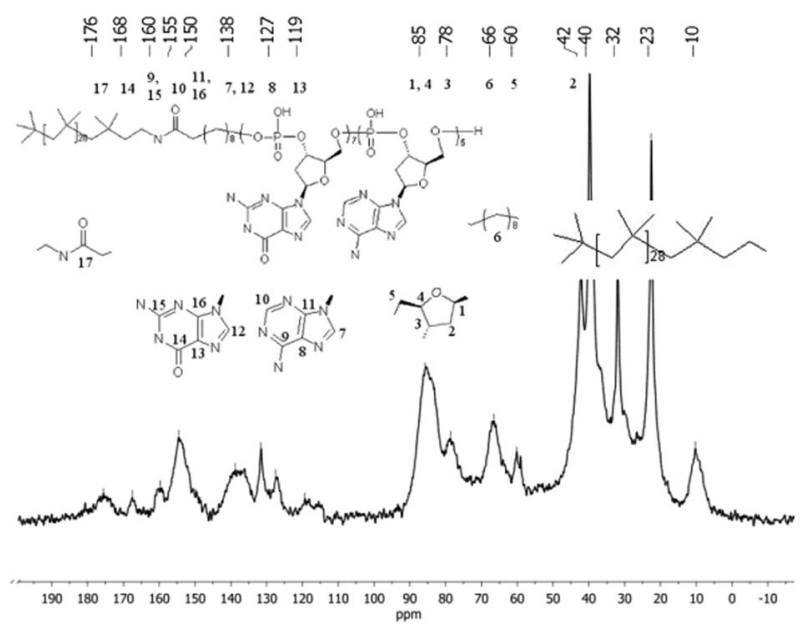

Fig. $1{ }^{13} \mathrm{C}$-CPMAS NMR of $\mathrm{PIB}_{31}-\mathrm{G}_{7} \mathrm{~A}_{5}$ : each peak can be assigned to a carbon-based group along the polymer backbone.

the chain configuration of the oligonucleotide (ESI $\dagger$, Fig. S3). The CD spectra of the nucleotide prior and subsequent to polymer modification with either PIB or PB are strictly identical. As expected, the wavelengths of the ellipticity maxima slightly shift with the composition of the nucleotide sequence. The minimum at $245 \mathrm{~nm}$ is typical of a B-form conformation of a DNA fragment corresponding to a compact cylindrical chain configuration of the nucleotide sequence. ${ }^{6,7}$ The amphiphilic nucleotide-based diblock copolymer can thus be modeled as a macromolecule consisting of a $4.08 \mathrm{~nm}$ long rigid rod covalently linked to a hydrophobic flexible polymer segment, with a Flory radius in a bad solvent of about $4 \mathrm{~nm} .{ }^{8}$ The full length of the selfassembled macromolecule is thus of about $8 \mathrm{~nm}$. The average diameter of spherical core-shell micelles should be $16 \mathrm{~nm}$ and, assuming fully stretched polymer and nucleotide sequences, of at least 26 and $45 \mathrm{~nm}$ for vesicles resulting from the self-assembly of PIB and PB modified nucleotide sequences, respectively.

Like with conventional self-assembling copolymers, the size and the size distribution of the self-assembled structures were reduced by sequential extrusion through 0.45 and $0.2 \mu \mathrm{m}$ membrane filters. The concentration reduction due to retention of some of the material in the filter membrane was controlled by UV spectroscopy. TEM and SEM of structures self-assembled from PIB modified nucleotide sequences reveal the presence of spherical objects with sizes ranging between 15 and $120 \mathrm{~nm}$ whereas the PB modification induces the self-assembly of slightly larger structures with sizes ranging between 20 and $150 \mathrm{~nm}$ (ESI $\dagger$, Fig. S4 for representative micrographs). In Fig. 2 is displayed a representative TEM obtained with self-assembled structures from PIB modified nucleotide sequences. The apparent hydrodynamic radii of the self-assembled structures measured by Dynamic Light Scattering (DLS, ESI $\dagger$, Fig. S5 for representative data) are $(236 \pm 9) \mathrm{nm}$, $(160 \pm 14) \mathrm{nm},(84 \pm 12) \mathrm{nm}$ and $(46 \pm 16) \mathrm{nm}$ for $\mathrm{PB}_{65}-\mathrm{A}_{5} \mathrm{G}_{7}$, $\mathrm{PIB}_{31}-\mathrm{G}_{7} \mathrm{~A}_{5}, \mathrm{PIB}_{31}-\mathrm{C}_{7} \mathrm{~T}_{5}$ and $\mathrm{PB}_{65}-\mathrm{T}_{5} \mathrm{C}_{7}$, respectively. Except for $\mathrm{PB}_{65}-\mathrm{T}_{5} \mathrm{C}_{7}$, the narrow size distribution indicates that well equilibrated structures are achieved. Larger sizes estimated by DLS in comparison to electron microscopy are due to the dry condition of the experimental set-up of electron microscopy. Additionally electrostatic interactions might arise due to insufficient screening by the residual salt concentration $(36 \mathrm{mM}$ as

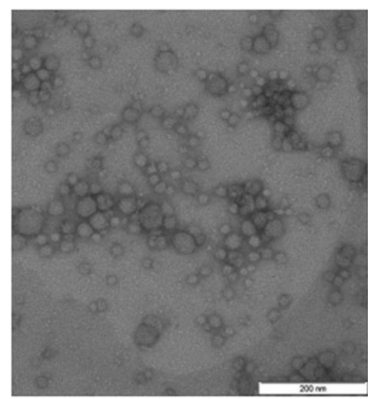

Fig. 2 Self-assembled $\mathrm{PIB}_{31}-\mathrm{G}_{7} \mathrm{~A}_{5}$ observed by TEM, white scale bar is $200 \mu \mathrm{m}$.

estimated by combined elementary analysis and UV spectroscopy) which leads to slower diffusion coefficients and therefore larger sizes quantification.

Few reports have been published to date on the structure formation and modes of interactions of polymer-modified nucleotide sequences ${ }^{4,5,9,10-14}$ and in particular on the formation of vesicular structures. This morphology appears to be favored by the physical incompatibility between the two blocks, i.e. coil synthetic hydrophobic polymer segment $v$ s. short nucleotide sequence, which engages in intermolecular interactions. Prior to closing to yield a vesicle, a sheet-like structure probably assembles. ${ }^{15,16}$ Light scattering investigations combined with electron microscopy corroborate this hypothesis. Owing to the size estimated by DLS, the self-assembled structures are likely vesicles. This assumption is further confirmed by the encapsulation experiments described in the following. Besides, comparison of the hydrodynamic radii of the structures self-assembled by $\mathrm{PB}_{65}-\mathrm{A}_{5} \mathrm{G}_{7}$ and $\mathrm{PB}_{65}-\mathrm{T}_{5} \mathrm{C}_{7}$ as well as $\mathrm{PIB}_{31}-\mathrm{G}_{7} \mathrm{~A}_{5}$ and $\mathrm{PIB}_{31}-\mathrm{C}_{7} \mathrm{~T}_{5}$ demonstrates that the composition of the nucleotide sequence influences the size, which decreases with lower $\mathrm{G}$ content. Reduced intermolecular interactions driven by $\pi-\pi$ stacking enabled by the $\mathrm{G}$ nucleotide probably hamper the formation of a membrane-like copolymer sheet prior to closing into vesicular structures. However, similar to the self-assembly of conventional synthetic block copolymers, a lower hydrophobic to hydrophilic ratio yields structures of smaller sizes. ${ }^{16}$

Morphological investigations and size estimations evidence that the polymer modified nucleotide sequences self-assemble into vesicular structures. The aqueous pool can therefore be loaded with a hydrophilic substance. To further assess the vesicular morphology, we encapsulated lactoperoxidase, which is an enzyme producing di-tyrosine, a fluorescent compound upon reaction of its tyrosine substrate in the presence of hydrogen peroxide. To demonstrate successful encapsulation, the permeability of the membrane needs to be tailored in order to ensure exchanges between the inner aqueous pool of the vesicular structure and the aqueous surrounding. To enable the enzymatic reaction, we resorted to the functional incorporation of pore-forming bacterial channel proteins in the hydrophobic polymer membrane of the vesicles. Diffusion of both the substrate of the enzyme and the product of the enzymatic reaction is thus ensured..$^{2}$ Self-assembly of the nucleotide-based block copolymer was therefore performed in the presence of the membrane protein in a solution of the enzyme. UV spectroscopy was carried out at each stage of the preparation procedure to assess the presence of the enzyme prior and subsequent to membrane filtration (ESI†, Fig. S6). 

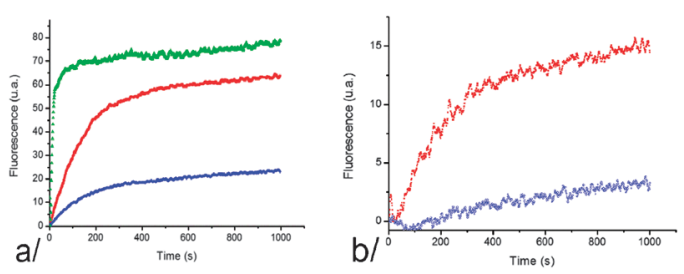

Fig. 3 Fluorescence spectroscopy (emission wavelength at $415 \mathrm{~nm}$ ) (a) represents the enzymatic activity of LPO at enzyme concentrations of 5 (green), 1 (red) and $0.5 \mu \mathrm{M}$ (blue). Graph (b) represents the comparative enzymatic activity of purified biological-like vesicles (red) and non-porous self-assemblies (blue).

In a subsequent step, centrifugal purification was performed to remove undesired compounds of low molecular weight. UV-vis spectroscopy of the self-assembled copolymer solution and the centrifuged fractions enabled us to assess both purity and concentration (ESI $\dagger$, Fig. S7). After five sequential centrifugation steps, a decreasing amount of polymer is centrifuged away until reaching a steady state concentration correlating with an eventually constant number of self-assembled structures. The free enzyme is cleared after three centrifugation steps. Since there is an overlap of the enzyme and oligonucleotide absorption peaks, the self-assembled structures are assumed purified when no enzymatic activity could be monitored by fluorescence spectroscopy in the centrifuged fraction (ESI $\dagger$, Fig. S8). The enzymatic activity of the centrifuged fraction is below the detection limit subsequent to three sequential purification steps, which is in agreement with comparative measurements performed with the solution of the free enzyme and the observation made by UV-vis spectroscopy. These characterizations are performed with selfassembled structures in the absence of the channel proteins as well. Besides, the vesicular structure provides protection of the encapsulated enzyme as assessed by activity measurement in a proteinase environment (ESI $\dagger$, Fig. S9). Overall, these investigations clearly demonstrate the efficient removal of nonencapsulated enzymes, the high purity of the system under investigation and the protective role of the nucleotide-based copolymer shell. Moreover, the UV sensitivity of the nucleic acids composing the self-assembling macromolecule enables the tracking of the concentration of the polymer-modified nucleotide sequence at any stage of the preparation procedure. Therefore combination of UV and fluorescence spectroscopy yields an accurate quantification of the encapsulation efficiency estimated to $12 \%$ of the initial enzyme concentration.

Measurements of the enzymatic activity through fluorescence spectroscopy at various enzyme concentrations $(0.5,1$ and $5 \mu \mathrm{M})$, keeping constant the tyrosine substrate concentration, enable us to conclude on the functional encapsulation of the enzyme (Fig. 3). Reaction speed and steady state values increase with increasing concentration of the enzyme. The enzymatic activity in the absence of channel proteins is comparable to background noise whereas, for the biological-like vesicles, activity could be estimated equivalent to a $0.5 \mu \mathrm{M}$ solution of the free enzyme. Further measurements were performed at various substrate concentrations (between 0.014 and $0.049 \mathrm{mg} \mathrm{mL}^{-1}$ ) to establish the MichaelisMentens kinetics of the system. ${ }^{17}$ The reaction rates $\left(K_{\mathrm{M}}\right)$ for both the encapsulated and free enzymes are of the same order of magnitude ( 253 and $344 \mu \mathrm{M}$ ) ensuring the native encapsulation of the enzyme since the substrate affinity is preserved.
The enzymatic turnover of this reaction of pseudo first order $\left(K_{\mathrm{cat}}\right)$ is however reduced by a factor of about $8\left(1.34 \mathrm{~s}^{-1}\right.$ vs. $\left.10.94 \mathrm{~s}^{-1}\right)$ upon encapsulation. Steric hindrance to activity between the substrate and the enzyme such as electrostatic interactions and hydrogen bonding might affect the enzymatic turnover raising an energetic barrier to enzymatic conversion of tyrosine to di-tyrosine. Diffusion limited transport through the channel protein of both the substrate and products of the enzymatic reaction also reduces the enzyme turnover, displacing the reaction equilibrium due to substrate deficiency and product excess in the confined inner pool of the vesicles.

Through co-assembly with native proteins, we demonstrated that the suitable polymer modification of nucleotide sequences yields vesicular structures. An enzyme is functionally encapsulated in the inner aqueous pool of the self-assembly whereas pore-forming proteins ensure diffusion of the substrate and product of the enzymatic reaction across the copolymer vesicular wall, otherwise not permeable. Similar to their biological analogues, the DNA-block copolymer vesicles achieve transport, enzyme storage and protection as well as activity. In combination with the hybridization property of the nucleotide sequence, this system therefore reveals several significant advantages for potential applications such as carriers for drug and gene delivery. Detailed structural characterizations are under progress to assess the modes of structure formation and interaction of this vesicle forming DNA block copolymers.

The SNSF (PPOOP2-128380), the University of Geneva and NCCR-NANO are greatly acknowledged for financial support. We especially thank W. Meier, G. Persy, S. Rigolet, L. Costato, J. Sonnet and A. Ziegler for scientific discussions and experimental support.

\section{Notes and references}

1 P. Tanner, S. Egli, V. Balasubramanian, O. Onaca, C. G. Palivan and W. Meier, FEBS Lett., 2011, 585, 1699-1706.

2 W. Meier, C. Nardin and M. Winterhalter, Angew. Chem., Int. Ed. 2000, 39, 4599-4602.

3 C. Nardin, S. Thoeni, J. Widmer, M. Winterhalter and W. Meier, Chem. Commun., 2000, 1433-1434.

4 F. E. Alemdaroglu and A. Herrmann, PMSE Prepr., 2007, 97, 968-970.

5 F. Teixeira Jr., P. Rigler and C. Vebert-Nardin, Chem. Commun., 2007, 1130-1132.

6 D. M. Hatters, L. Wilson, B. W. Atcliffe, M. T. D. N. GuzzoPernell and G. Howlett, Biophys. J., 2001, 81, 371-381.

7 J. Kypr, I. Kejnovska, D. Renciuk and M. Vorlickova, Nucleic Acids Res., 2009, 37, 1713-1725.

8 P. C. Hiemenz and T. P. Lodge, Polymer Chemistry, CRC Press, Boca Raton, 2007.

9 F. E. Alemdaroglu, M. Safak, J. Wang, R. Berger and A. Herrmann, Chem. Commun., 2007, 1358-1359.

10 F. E. Alemdaroglu and A. Herrmann, Org. Biomol. Chem., 2007, 5, $1311-1320$.

11 F. E. Alemdaroglu, N. C. Alemdaroglu, P. Langguth and A. Herrmann, Polymer Prepr., 2007, 48, 1033-1034.

12 A. Herrmann, F. E. Alemdaroglu, M. Safak and E. Ergen, Polymer Prepr., 2007, 48, 994-995.

13 N. Cottenye, F. Teixeira Jr., A. Ponche, G. Reiter, K. Anselme, W. Meier, L. Ploux and C. Vebert-Nardin, Macromol. Biosci., $2008,8,1161-1172$.

14 L. Caseli, C. P. Pascholati, F. Teixeira, S. Nosov, C. Vebert, A. H. E. Müeller and O. N. Oliveira, J. Colloid Interface Sci., 2010, 347, 56-61.

15 H. Kukula, H. Schlaad, M. Antonietti and S. Förster, J. Am. Chem. Soc., 2002, 124, 1658-1663.

16 D. E. Discher and A. Eisenberg, Science, 2002, 297, 967-973.

17 H. Lee and I. Wilson, Biochim. Biophys. Acta, Enzymol., 1971, 242, $519-522$. 